\title{
Meal-Induced Acceleration of Tablet Transit Through the Human Small Intestine
}

\author{
Hala M. Fadda, ${ }^{1}$ Emma L. McConnell, ${ }^{1}$ Michael D. Short, ${ }^{2}$ and Abdul W. Basit ${ }^{1,3}$
}

Published online January 10, 2009

The following error was published in the article:

The second sentence of the CONCLUSIONS:

It should read 'Administration of tablets 45 min before food resulted in an increased small intestinal transit over fasted and fed doses', instead of 'Administration of tablets $45 \mathrm{~min}$ before food resulted in an increased small intestinal transit time over fasted and fed doses'. The word 'time' should be removed from the sentence.

\footnotetext{
${ }^{1}$ Department of Pharmaceutics, The School of Pharmacy, University of London, 29-39 Brunswick Square, London, WC1N 1AX, UK.

${ }^{2}$ Department of Medical Physics and Bioengineering, University College London Hospitals, Capper Street, London, WC1E 6JA, UK.

${ }^{3}$ To whom correspondence should be addressed. (e-mail: abdul.basit@pharmacy.ac.uk)
}

The online version of the original article can be found at: http://dx.doi.org/10.1007/s11095-008-9749-2 